\title{
Fibroblast growth factor receptor isotype expression and its association with overall survival in patients with hepatocellular carcinoma
}

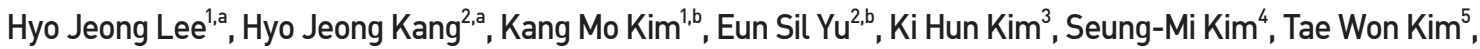 \\ Ju Hyun Shim', Young-Suk Lim', Han Chu Lee', Young-Hwa Chung', and Yung Sang Lee ${ }^{1}$ \\ ${ }^{1}$ Department of Internal Medicine, Asan Liver Center, ${ }^{2}$ Department of Pathology, ${ }^{3}$ Division of Hepatobiliary Surgery and Liver \\ Transplantation, Department of Surgery, ${ }^{4}$ Innovative Cancer Research, Asan Institute for Life Science and ${ }^{5}$ Department of Oncology, \\ Asan Medical Center, University of Ulsan College of Medicine, Seoul, Korea
}

Background/Aims: Fibroblast growth factor signaling is involved in hepatocarcinogenesis. The aim of this study was to determine the fibroblast growth factor receptor (FGFR) isotype expression in hepatocellular carcinoma (HCC) and neighboring nonneoplastic liver tissue, and elucidate its prognostic implications.

Methods: Immunohistochemical staining of FGFR1, $-2,-3$, and -4 was performed in the HCCs and paired neighboring nonneoplastic liver tissue of $870 \mathrm{HCC}$ patients who underwent hepatic resection. Of these, clinical data for 153 patients who underwent curative resection as a primary therapy were reviewed, and the relationship between FGFR isotype expression and overall survival was evaluated (development set). This association was also validated in 73 independent samples (validation set) by Western blot analysis.

Results: FGFR1, $-2,-3$, and -4 were expressed in $5.3 \%, 11.1 \%, 3.8 \%$, and $52.7 \%$ of HCCs, respectively. Among the development set of 153 patients, FGFR2 positivity in HCC was associated with a significantly shorter overall survival (5-year survival rate, $35.3 \%$ vs. $61.8 \% ; P=0.02$ ). FGFR2 expression in HCC was an independent predictor of a poor postsurgical prognosis (hazard ratio, 2.10; $P=0.02$ ) in the development set. However, the corresponding findings were not statistically significant in the validation set.

Conclusions: FGFR2 expression in HCC could be a prognostic indicator of postsurgical survival. (Clin Mol Hepatol 2015;21:60-70)

Keywords: Hepatocellular carcinoma; Fibroblast growth factor; Receptor; Immunohistochemistry; Survival

\section{Abbreviations:}

HCC, hepatocellular carcinoma; FGFR, fibroblast growth factor receptor; FGF, fibroblast growth factor; YSR, year survival rate; AJCC, American Joint Committee on Cancer

${ }^{a}$ These two authors contributed equally to this work.

${ }^{\mathrm{b}}$ These two authors are co-corresponding authors.

\author{
Corresponding author : Kang Mo Kim \\ Department of Internal Medicine, Asan Liver Center, Asan Medical Center, \\ University of Ulsan College of Medicine, 88 Olympic-ro 43-gil, Songpa-gu, \\ Seoul 138-736, Korea \\ Tel: +82-2-3010-3190, Fax: +82-2-485-5782 \\ E-mail: kimkm70@amc.seoul.kr \\ Eun Sil Yu \\ Department of Pathology, Asan Medical Center, University of Ulsan College \\ of Medicine, 88 Olympic-ro 43-gil, Songpa-gu, Seoul 138-736, Korea \\ Tel: +82-2-3010-4552, Fax: +82-2-485-8121 \\ E-mail:esyu@amc.seoul.kr
}


Hyo Jeong Lee, et al. FGFR2 expression predicts survival in HCC

\section{INTRODUCTION}

Hepatocellular carcinoma (HCC) is the third most common cause of cancer-related death worldwide and its incidence is increasing. ${ }^{1,2}$ In patients in the early stages HCC, hepatic resection is the treatment of choice, but even after curative resection, recurrence is common, and is the main cause of death. ${ }^{1,3}$ However, there are no adequate adjuvant therapies for HCC patients who have undergone curative hepatic resection except interferon. ${ }^{4}$ Moreover, most HCC patients are diagnosed at advanced stages, and the prognosis for advanced HCC is currently very poor. ${ }^{5,6}$ The only effective systemic agent is sorafenib, which has shown modest effects in prolonging survival. ${ }^{7}$ The progression of HCC from preneoplastic lesions to advanced HCC occurs stepwise. Therefore, investigation into the disturbed signaling pathways relative to hepatocarcinogenesis could provide invaluable information in the development of new preventive, and therapeutic options. ${ }^{8}$

Fibroblast growth factor (FGF)/ FGF receptor (FGFR) signaling has been reported to be involved in the progression of many cancers $^{9}$ and there is also increasing evidence for the role of FGF signaling in hepatocarcinogenesis. ${ }^{10-13}$ FGF/FGFR signaling has evolved to become a highly complex growth factor signaling pathway. The mammalian FGF family comprises 18 ligands, which exert their actions through four highly conserved transmembrane tyrosine kinase receptors (FGFR1, FGFR2, FGFR3, and FGFR4). ${ }^{9}$ There have been several studies that have focused on individual FGFR isotypes in HCC. However, the expression patterns of multiple FGFR isotypes in HCC and in neighboring liver tissues have not been fully evaluated other than in one small study. ${ }^{14}$ Moreover, although there have been several studies of the potential role of each FGFR isotype in hepatocarcinogenesis, ${ }^{10-13}$ the reported results are inconsistent. Hence, the FGFR isotype expression pattern
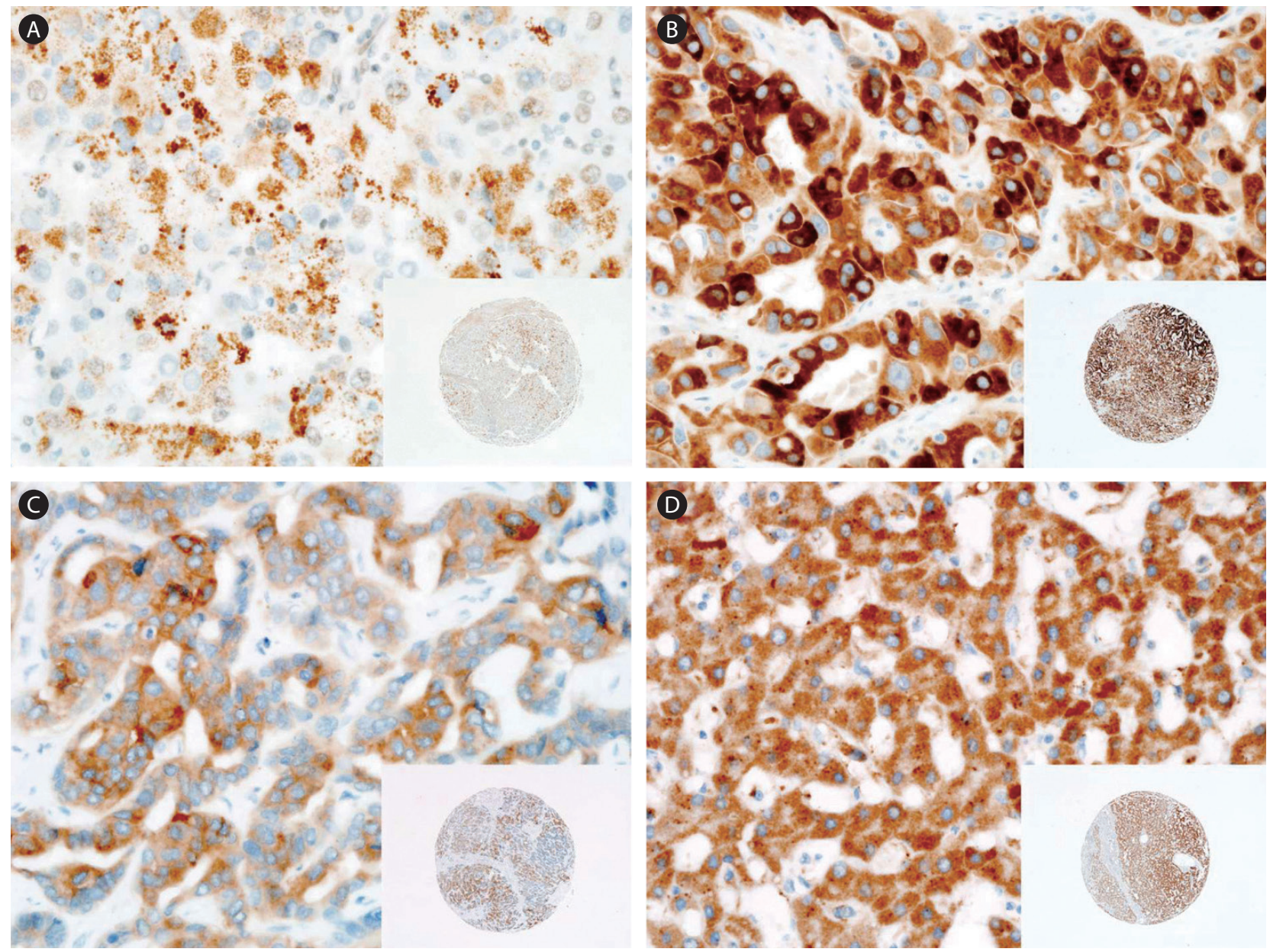

Figure 1. Representative immunostaining results for FGFR1-4 in HCC tissue. (A) FGFR1 staining showing weak and focal expression. (B) FGFR2 staining showing strong and diffuse expression. (C) FGFR3 staining showing weak-to-moderate and diffuse expression. (D) FGFR4 staining showing strong and diffuse expression. Original magnifications, $\times 400$ and $\times 40$ (insets). 
in human HCC and its association with the clinical outcomes of this disease remains still unclear.

The aims of our present study were to assess the FGFR isotype expression pattern in HCC and neighbouring non-neoplastic liver from resected human tissues and elucidate its prognostic implications.

\section{MATERIALS AND METHODS}

\section{Study population and validation set}

In total, 870 HCC patients who had undergone surgical resection at the Asan Medical Center between 1998 and 2004 were evaluated for FGFR isotype expression. Of these, the $153 \mathrm{HCC}$ patients who had received a curative resection as a primary therapy between 2003 and 2004 were selected for assessing clinical outcome (development set, Supplemental Fig. 1). A total of 267 cases received a curative resection in that period. We excluded 99 cases due to other treatments before hepatic resection, 12 cases who were diagnosed with double primary cancer, and three cases due to incomplete data. The clinical data for the remaining $153 \mathrm{pa}$ tients were reviewed, and the association between FGFR isotype expression and overall survival were evaluated. In this development set, 130 patients (85.0\%) were male and the median age was 52 years (range: 17-80 years). The differences in the baseline clinicopathological characteristics according to each FGFR isotype expression pattern in HCC tissues are described in Table 1 and Supplemental Table 1. FGFR3 and 4 positivity was observed more frequently in male HCC patients $(P=0.01$ and $P=0.03$, respectively), other than that, there were no significant clinicopathological differences found according to each FGFR isotype expression.

Table 1. Comparison of the baseline characteristics between the FGFR2- and FGFR4-positive and -negative groups in HCC tissue

\begin{tabular}{|c|c|c|c|c|c|c|}
\hline \multirow{2}{*}{ Variables } & \multicolumn{2}{|c|}{ FGFR2 expression in HCC } & \multirow{2}{*}{$P$-value } & \multicolumn{2}{|c|}{ FGFR4 expression in HCC } & \multirow{2}{*}{$P$-value } \\
\hline & Positive $(n=17)$ & Negative $(n=136)$ & & Positive $(n=71)$ & Negative $(n=82)$ & \\
\hline Age, years, median (range) & $53(34-78)$ & $52(17-80)$ & 0.61 & $51(17-79)$ & $52(31-78)$ & 0.97 \\
\hline Sex, male, no. (\%) & $13(76.5)$ & $117(86.0)$ & 0.29 & $65(91.5)$ & $65(79.3)$ & 0.03 \\
\hline \multicolumn{7}{|l|}{ Etiology, no. (\%) } \\
\hline HBV & $15(88.2)$ & $120(88.2)$ & 0.77 & $65(91.5)$ & $70(85.4)$ & 0.29 \\
\hline $\mathrm{HCV}$ & $0(0.0)$ & $9(6.6)$ & & $3(4.2)$ & $6(7.3)$ & \\
\hline Alcohol & $2(11.8)$ & $4(2.9)$ & & $2(2.8)$ & $4(4.9)$ & \\
\hline Others & $0(0.0)$ & $3(2.2)$ & & $1(1.4)$ & $2(2.4)$ & \\
\hline \multicolumn{7}{|l|}{ Child-Pugh class, no. (\%) } \\
\hline$A$ & $15(88.2)$ & $126(92.6)$ & 0.63 & $64(90.1)$ & $77(93.9)$ & 0.39 \\
\hline B & $2(11.8)$ & $10(7.4)$ & & $7(9.9)$ & $5(6.1)$ & \\
\hline \multicolumn{7}{|c|}{ Serum alpha fetoprotein, no. (\%) } \\
\hline$>400 \mathrm{ng} / \mathrm{mL}$ & $8(47.1)$ & $47(34.6)$ & 0.31 & $26(36.6)$ & $29(35.4)$ & 0.87 \\
\hline \multicolumn{7}{|c|}{ Intrahepatic HCC morphology, no. (\%) } \\
\hline Nodular type & $16(94.1)$ & $133(97.8)$ & 0.38 & $70(98.6)$ & $79(96.3)$ & 0.62 \\
\hline Infiltrative type & $1(5.9)$ & $3(2.2)$ & & $1(1.4)$ & $3(3.7)$ & \\
\hline \multicolumn{7}{|l|}{ T stage, no.(\%) } \\
\hline 1 & $12(70.6)$ & $96(70.6)$ & 0.53 & $55(77.5)$ & $53(64.6)$ & 0.21 \\
\hline 2 & $2(11.8)$ & $30(22.1)$ & & $10(14.1)$ & $22(26.8)$ & \\
\hline 3 & $3(17.6)$ & $10(7.4)$ & & $6(8.5)$ & $7(8.5)$ & \\
\hline Portal vein invasion, no. (\%) & $1(5.9)$ & $4(2.9)$ & 0.45 & $2(2.8)$ & $3(3.7)$ & 1.00 \\
\hline \multicolumn{7}{|c|}{ Edmondson-Steiner grade, no. (\%) } \\
\hline I & $0(0.0)$ & $5(3.7)$ & 0.68 & $3(4.3)$ & $2(2.5)$ & 0.44 \\
\hline$\|$ & $5(29.4)$ & $42(31.3)$ & & $22(31.4)$ & $25(30.9)$ & \\
\hline III & $11(64.7)$ & $76(56.7)$ & & $41(58.6)$ & $46(56.8)$ & \\
\hline IV & $1(5.9)$ & $11(8.2)$ & & $4(5.7)$ & $8(9.9)$ & \\
\hline
\end{tabular}

FGFR, fibroblast growth factor receptor; HCC, hepatocellular carcinoma; HBV, hepatitis B virus; HCV, hepatitis C virus. 
Hyo Jeong Lee, et al. FGFR2 expression predicts survival in HCC

Table 2. Immunohistochemical staining methods used to detect the expressions of FGFR1-4

\begin{tabular}{lcll}
\hline Type & Dilution & \multicolumn{1}{c}{ Antibodies } & \multicolumn{1}{c}{ Control } \\
\hline FGFR1 & $1: 200$ & Rabbit, polyclonal, Abnova, Taipei City, Taiwan (cat. PAB17005) & Lung: adenocarcionma \\
FGFR2 & $1: 200$ & Mouse, monoclonal, Abnova, Taipei City, Taiwan (cat. H00002263-M01, clone 1G3) & Stomach: adenocarcinoma \\
FGFR3 & $1: 50$ & Mouse, monoclonal, Santa Cruz Biotechnology, CA, USA (cat. SC-13121, clone B-9) & Skin: normal epidermis \\
FGFR4 & $1: 35$ & Rabbit, polyclonal, Santa Cruz Biotechnology, CA, USA (cat. SC-124, clone C-16) & $\begin{array}{c}\text { Breast: invasive ductal carcinoma / } \\
\text { prostate: adenocarcinoma }\end{array}$ \\
\hline
\end{tabular}

FGFR, fibroblast growth factor receptor.

Table 3. Frequencies of FGFR isotype positivity in HCC and neighboring liver tissue from 870 HCC patients who underwent hepatic resection between 1998 and 2004 (study population), and in 153 HCC patients who underwent curative hepatic resection as a primary treatment between 2003 and 2004 (development set)

\begin{tabular}{|c|c|c|c|c|c|c|}
\hline & \multicolumn{2}{|r|}{$\mathrm{n}=870$} & \multirow{2}{*}{$P$-value } & \multicolumn{2}{|r|}{$n=153$} & \multirow{2}{*}{$P$-value } \\
\hline & HCC (\%) & Neighboring liver tissue (\%) & & HCC (\%) & Neighboring liver tissue (\%) & \\
\hline FGFR1 & $46(5.3)$ & $52(5.9)$ & 0.61 & $4(2.6)$ & $14(9.2)$ & 0.02 \\
\hline FGFR2 & $97(11.1)$ & $0(0.0)$ & $<0.01$ & $17(11.1)$ & $0(0.0)$ & $<0.01$ \\
\hline FGFR3 & $33(3.8)$ & $0(0.0)$ & $<0.01$ & $4(2.6)$ & $0(0.0)$ & 0.13 \\
\hline FGFR4 & $458(52.7)$ & $721(82.9)$ & $<0.01$ & $71(46.4)$ & 135 (88.2) & $<0.01$ \\
\hline
\end{tabular}

FGFR, fibroblast growth factor receptor; HCC, hepatocellular carcinoma.

To validate our FGFR isotype expression analysis and its clinical implications, we selected an additional $73 \mathrm{HCC}$ patients (56 males [76.7\%]; median age, 55 years [range: 28-79 years], Supplemental Table 2) obtained from an independent cohort which included HCC patients who had received a curative resection as their primary therapy between 2007 and 2009. In this validation set, we performed western blotting of FGFR2 and also validated the association between FGFR2 expression and overall survival were evaluated. There were no significant clinicopathological differences found between the patients with FGFR2-positive or -negative HCC (Supplemental Table 3).

This study protocol was approved by the institutional review board of the Asan Medical Center (protocol no. 2007-0332 and 2011-0115) in compliance with the Declaration of Helsinki. Informed consent was not obtained, because the data were retrospectively collected after the completion of treatment. Patient records/information was anonymized and de-identified prior to analysis.

\section{Tissue microarray construction}

For FGFR isotype expression analysis, we established 55 tissue microarray slides using formalin-fixed paraffin-embedded tissues from our 870 surgically resected HCC and neighboring non-neoplastic liver tissue samples. Representative areas of tumors were marked on hematoxylin and eosin-stained slides. Duplicates of two cores from the tumor tissue and one core from the non-neo- plastic liver tissue, measuring $1.5 \mathrm{~mm}$ in diameter, were arrayed from the corresponding paraffin blocks into a recipient block using an arraying machine (Tissue microarrayer; Pathology Devices, Westminster, MD, USA). The first $4 \mu \mathrm{m}$ sections from these arrays were examined for validation purposes, and additional sections were used for immunohistochemistry.

\section{Immunohistochemistry}

Immunohistochemical staining for FGFR1-4 was performed using tissue microarray slides with the Ventana Benchmark automated staining system (Ventana Medical Systems, Tucson, AZ, USA). The primary antibody against FGFR 1-4 are indicated in Table 2. Tissue sections of $4 \mu \mathrm{m}$ were transferred onto poly-L-lysine-coated adhesive slides and dried at $62^{\circ} \mathrm{C}$ for 30 minutes. Antigens were retrieved by heating the sections for one hour in $\mathrm{CC} 1$ diamine tetraacetic acid, $\mathrm{pH}$ 8.0. After primary antibody incubation for 32 minutes at $37^{\circ} \mathrm{C}$, detection was carried out with the Ventana UItraview DAB detection kit.

\section{Evaluation of immunohistochemistry results}

All slides were independently evaluated by two pathologists who co-authored this study (EY, HJK). The expression profiles of FGFR2 and FGFR4 were scored according to staining intensity (0, negative; 1 , weak; 2 , moderate; 3 , strong) and the proportion of 
positively stained cells. The HCC cases were considered positive for FGFR2 and FGFR4 when the staining intensity was moderate to strong and the proportion of positively stained cells was greater than $10 \%$. The expression profiles of FGFR1 and FGFR3 were only scored according to their proportion of positive cells, because the staining intensities for FGFR1 and FGFR3 were weak to moderate and the proportion of positive cells was low for these isotypes. The $\mathrm{HCC}$ cases were considered positive for FGFR1 and FGFR3 when the proportion of positively stained cell was greater than $10 \%$.

\section{Western blotting}

The 73 HCC and neighboring liver tissue samples in the validation set, which were stored in liquid nitrogen, were homogenized in chilled lysis buffer (1X RIPA buffer, Cell Signaling Technology Inc., Beverly, MA, USA), and then placed on ice for 20 min. After centrifugation at $13,000 \mathrm{rpm}$ for $20 \mathrm{~min}$ at $4^{\circ} \mathrm{C}$, the supernatants were collected as protein samples. Protein contents were measured using a Bradford assay, resolved on SDS-PAGE gels and transferred to polyvinylidene difluoride membranes. After blockage of non-specific binding sites for $1 \mathrm{~h}$ with $5 \%$ skim milk diluted in Tris buffered saline with Tween 20, the polyvinylidene difluoride membrane was incubated overnight at $4^{\circ} \mathrm{C}$ with primary antibodies (anti-FGFR2 antibody; Abnova, Taipei city, Taiwan), and then rinsed 3 times by 10 mins with Tris buffered saline with Tween 20 . The membrane was then incubated further with HRP-conjugated secondary antibodies at room temperature, and then washed three times with $0.15 \%$ Tris buffered saline with Tween 20 . Signals on these immunoblots were detected using the ECL kit (Amersham Life Sciences Inc., Buckinghamshire, UK).
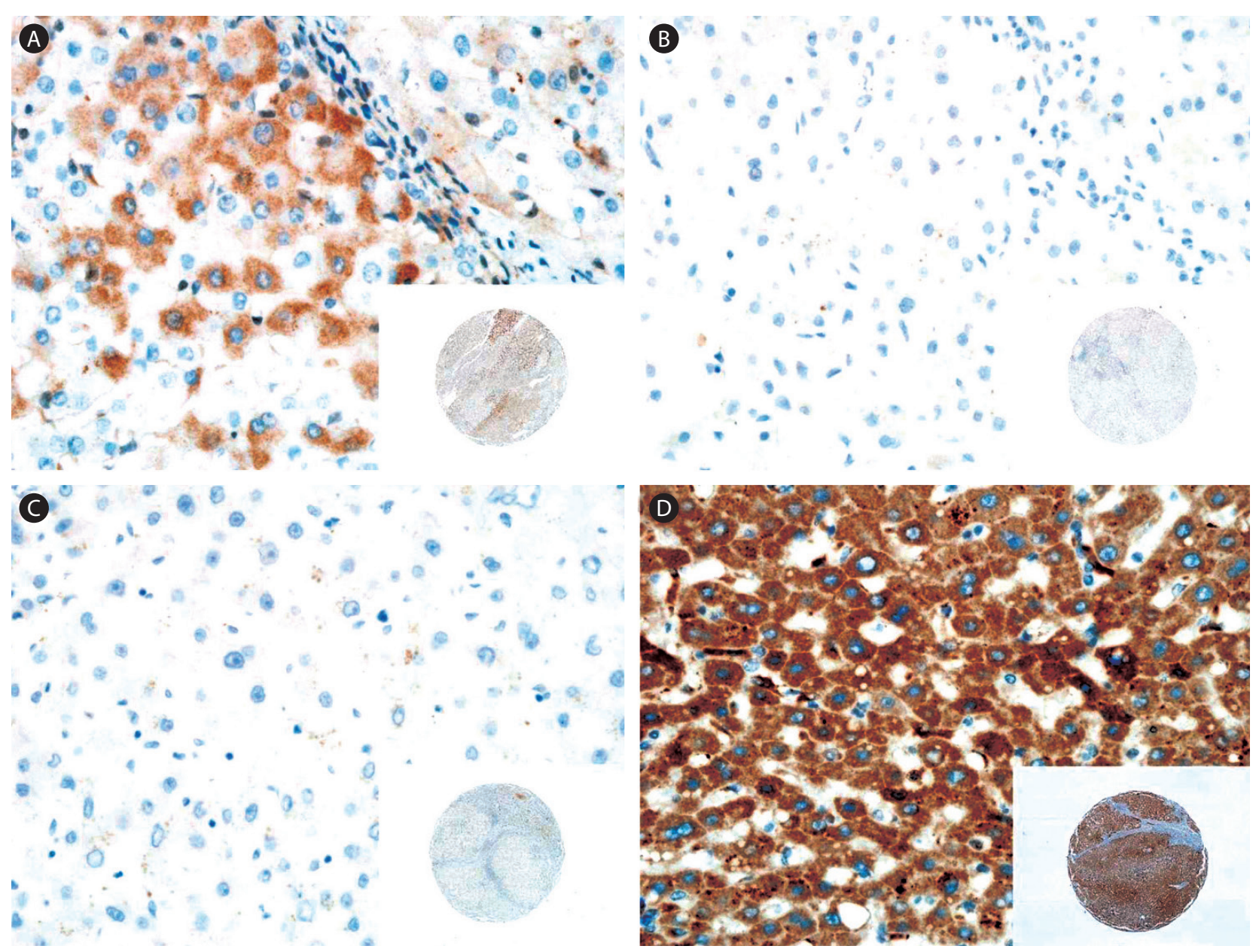

Figure 2. Representative immunostaining results for FGFR1-4 in nonneoplastic liver tissue neighboring the HCC tumor tissue. (A) FGFR1 staining showing weak and focal expression in the cytoplasm. (B) No signal for FGFR2 and -3. (D) FGFR4 staining showing strong and diffuse expression in the cytoplasm. Original magnifications, $\times 400$ and $\times 40$ (insets). 
Hyo Jeong Lee, et al. FGFR2 expression predicts survival in HCC

\section{Statistical analysis}

Differences in continuous variables between two groups were evaluated using the Student $t$-test, and differences in categorical variables were evaluated with the $X^{2}$ test or Fisher's exact test. McNemar's test was used to evaluate differences in matched pairs with a dichotomous trait. The Kaplan-Meier method was used to calculate and display overall survival curves, and the log-rank test was performed to determine differences among all groups. Univariate prognostic factors were entered into a Cox proportional hazards model using stepwise selection to identify independent predictors of death. Statistical significance was defined by a $P$-value of $<0.05$. The SPSS statistical software (version 18.0; SPSS, Chicago, IL, USA) was used for all analyses.

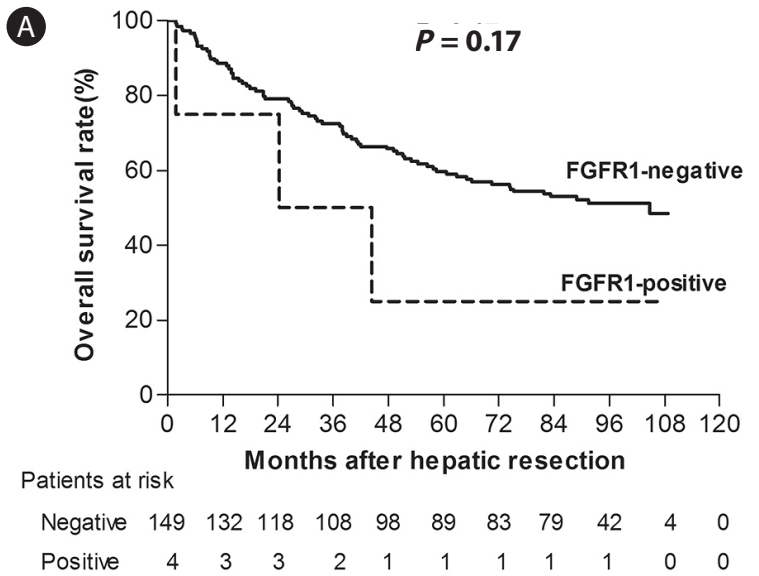

C

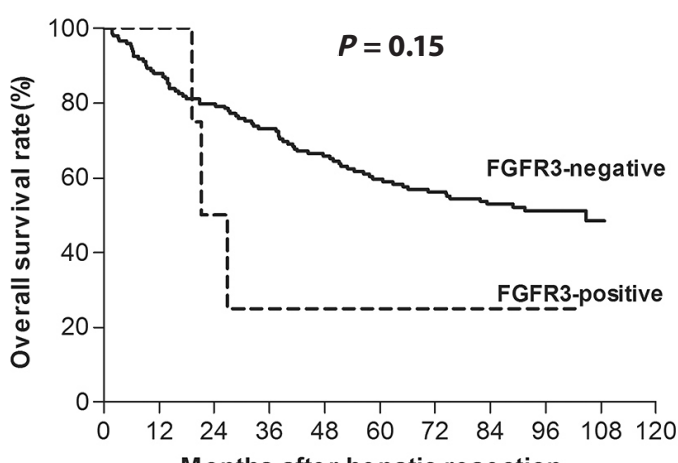

Patients at risk

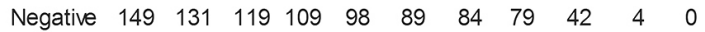
$\begin{array}{llllllllllll}\text { Positive } & 4 & 4 & 2 & 1 & 1 & 1 & 1 & 1 & 1 & 0 & 0\end{array}$

\section{RESULTS}

\section{FGFR isotype expression patterns in HCC and neighboring liver tissues}

Immunohistochemical staining analysis for FGFR1-4 in HCC and neighboring liver tissues is shown in Figure 1 and 2, respectively. The expression of all four FGFR isotypes in HCC cells was found to be cytoplasmic (Fig. 1). Neighboring non-neoplastic hepatocytes also showed cytoplasmic expression of FGFR1 and 4 in some cases (Fig. 2A, D), but FGFR2- and FGFR3-positive cells were not detected in non-neoplastic liver tissues (Fig. 2B, 2C).

The frequencies of FGFR isotype expression in all 870 samples in the study cohort are indicated in Table 3. FGFR1 expression was observed in a small number of both HCC (5.3\%) and neighboring liver tissues (5.9\%). FGFR2 and 3 expression was observed only in

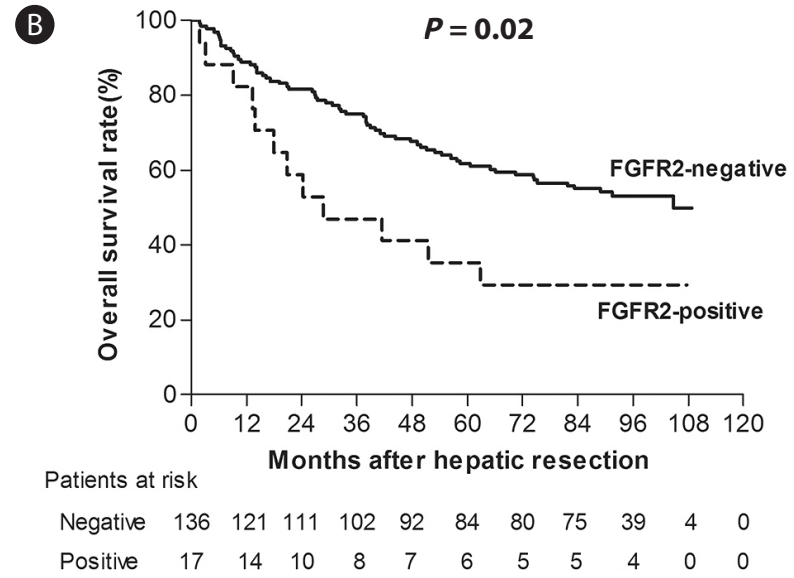

(D)

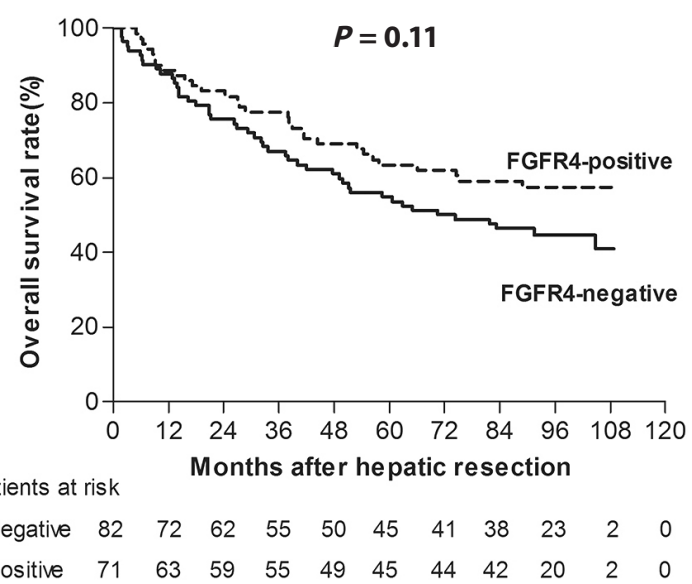

Figure 3. Kaplan-Meier survival estimates according to the expression levels of (A) FGFR1, (B) FGFR2, (C) FGFR3, and (D) FGFR4 in HCC tissue (development set). 
HCC tissues (18.7 and 3.8\%, respectively). FGFR4 expression was observed most frequently in both HCC (52.9\%) and neighboring liver tissues (82.9\%). There was a statistically significant difference in the expression levels of FGFR2, 3 and 4 between HCC and neighboring liver tissue. The frequencies of FGFR isotype expression in the 153 patients for whom clinical data were available (development set), are listed in Table 3. FGFR1 expression was significantly higher in neighboring liver tissues (9.2\%) than in HCC (2.6\%). FGFR3 expression in HCC tissues was observed less frequently than 870 full study population (2.6\%). FGFR2 and FGFR4 isotype expression patterns of the 153 samples in our clinical data appeared to be grossly similar to those in the full 870 sample.

\section{Patients with FGFR2-positive HCC show a significantly poorer overall survival outcome}

We generated Kaplan-Meier survival curves of 153 HCC patients (development set) who we stratified for each FGFR isotype expression (Fig. 3). FGFR2-positive HCC patients showed a poorer overall survival rate than FGFR2-negative cases and this difference was found to be statistically significant (5 year survival rate (YSR), $35.3 \%$ vs. $61.8 \% ; P=0.02 ;$ Fig. $3 \mathrm{~B})$.

The overall survival rates of the patients with FGFR1-positive HCC were poorer than FGFR1-negative cases (5 YSR of $25.0 \%$ vs.
$59.7 \%)$, but as only four of our patients showed FGFR1-positive HCC, this was not statistically significant ( $P=0.17$; Fig. $3 A)$. The 5 YSR of the patients with FGFR3-positive or -negative HCC was $25.0 \%$ and $59.7 \%$, respectively, but as found for FGFR1, the number FGFR3-positive HCC cases was only 4 and the difference was again not significant $(P=0.16$; Fig. $3 C$ ). In addition, the overall survival rates of the patients with FGFR4-positive or -negative HCC showed no statistical difference (5 YSR 63.4\% vs. 54.9\%, $P=0.11$; Fig. 3D).

\section{FGFR2 expression in HCC is an independent predictor of a poor postsurgical prognosis}

Table 4 lists the results of our univariate and multivariate analysis of the $153 \mathrm{HCC}$ patients to identify FGFR isotypes that are possible prognostic factors. These analyses thus included clinicopathologic characteristics (i.e., age, sex, Child-Pugh class, serum alpha fetoprotein, HCC morphology, American Joint Committee on Cancer (AJCC) T stage, portal vein invasion, Edmondson-Steiner grade) and the expression status of each FGFR isotype. Univariate analysis using a Cox regression model showed that a female sex $(P=0.01)$, Child-Pugh class B $(P<0.01)$, infiltrative type HCC $(P=0.04)$, T3 stage $(P<0.01)$ and positive FGFR2 expression of HCC ( $P=0.02)$ as significant prognostic markers for HCC compared with the relevant baseline conditions. Multivariate analysis using a

Table 4. Univariate and multivariate analyses of the prognostic factors affecting the survival of HCC patients in the development set

\begin{tabular}{|c|c|c|c|c|}
\hline \multirow{2}{*}{ Variables } & \multicolumn{2}{|l|}{ Univariate } & \multicolumn{2}{|l|}{ Multivariate } \\
\hline & Hazard ratio $(95 \% \mathrm{Cl})$ & $P$ value & Hazard ratio $(95 \% \mathrm{Cl})$ & $P$ value \\
\hline Age, $\geq 60$ vs $<60$ years & $1.52(0.94-2.47)$ & 0.09 & & \\
\hline Sex, male vs female & $0.49(0.28-0.85)$ & 0.01 & $0.55(0.31-0.99)$ & 0.05 \\
\hline Child-Pugh class, B vs A & $3.02(1.54-5.89)$ & $<0.01$ & $2.97(1.42-6.21)$ & $<0.01$ \\
\hline Serum alpha fetoprotein, $>400 \mathrm{vs} \leq 400 \mathrm{ng} / \mathrm{mL}$ & $1.13(0.71-1.82)$ & 0.59 & & \\
\hline HCC morphology, infiltrative vs nodular & $3.38(1.06-10.75)$ & 0.04 & & \\
\hline AJCC T stage, 3 vs 1-2 & $3.59(1.89-6.84)$ & $<0.01$ & $3.05(1.52-6.12)$ & $<0.01$ \\
\hline Portal vein invasion, present vs absent & $2.69(0.98-7.38)$ & 0.05 & & \\
\hline Edmondson-Steiner grade, III-IV vs I-II & $1.45(0.88-2.39)$ & 0.15 & & \\
\hline FGFR1 expression of HCC, positive vs negative & $2.19(0.69-6.97)$ & 0.18 & & \\
\hline $\begin{array}{l}\text { FGFR1 expression of neighboring liver tissue, } \\
\text { positive vs negative }\end{array}$ & $1.03(0.47-2.24)$ & 0.95 & & \\
\hline FGFR2 expression of HCC, positive vs negative & $2.07(1.12-3.85)$ & 0.02 & $2.10(1.11-3.98)$ & 0.02 \\
\hline FGFR3 expression of HCC, positive vs negative & $2.24(0.70-7.13)$ & 0.17 & & \\
\hline FGFR4 expression of HCC, positive vs negative & $0.69(0.43-1.09)$ & 0.11 & & \\
\hline $\begin{array}{l}\text { FGFR4 expression of neighboring liver tissue, positive vs } \\
\text { negative }\end{array}$ & $0.95(0.38-2.36)$ & 0.91 & & \\
\hline
\end{tabular}

HCC, hepatocellular carcinoma ; Cl, confidence interval; AJCC, American Joint Committee on Cancer; FGFR, fibroblast growth factor receptor. 
Hyo Jeong Lee, et al. FGFR2 expression predicts survival in HCC

Cox regression model on the same set of patients identified ChildPugh class B $(P<0.01)$, AJCC T3 stage $(P=0.02)$, and positive FGFR2 expression of HCC $(P=0.02)$ as independent predictors of a poor prognosis in terms of postsurgical survival of HCC patients.

\section{Validation of the FGFR2 analyses in an independent cohort}

We analyzed a cohort of 73 additional HCC patients to validate our FGFR2 data and their clinical implications for HCC patients. FGFR2 expression was measured by western blotting in this validation set (Supplemental Fig. 2) and was detected in $28.8 \%$ of the HCC tissue samples. Survival analysis showed a shorter overall survival trend in the FGFR2-positive HCC patients in the validation set (5 YSR, 49.7\% vs. $71.2 \%$ ) but this failed to reach clinical significance ( $P=0.19$; Fig. 4). In multivariate analysis to identify prognostic factors for HCC, the hazard ratio of positive FGFR2 expression in HCC was 2.18 and showed borderline significance $(P=0.07)$.

\section{DISCUSSION}

The present study demonstrated, for the first time, FGFR isotype expression patterns in HCC and neighbouring non-neoplastic liver tissue in a large HCC patient cohort. The notable findings were that FGFR2 expression was observed only in human HCC tissue (18.7\%), but not in neighboring non-neoplastic liver tissue, suggesting that hepatocytes might express high levels of FGFR2 under hepatocarcinogenesis conditions. Positive FGFR2 expression in

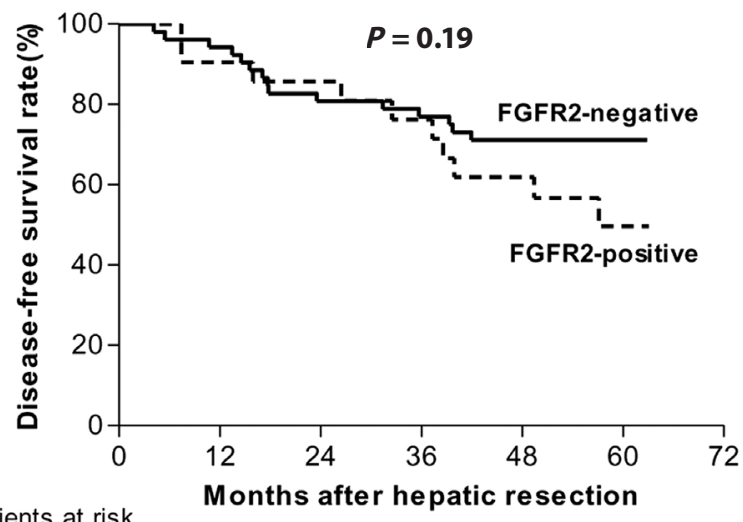

Patients at risk

$\begin{array}{lllllll}\text { Negative } & 52 & 49 & 42 & 40 & 37 & 10 \\ \text { Positive } & 21 & 19 & 18 & 16 & 13 & 5\end{array}$

Figure 4. Kaplan-Meier survival estimates according to the FGFR2 expression levels in HCC tissue (validation set).
HCC tissues was an independent prognostic factor for postsurgical survival in HCC patients in the large cohort, though this finding was not proved in the validation set.

FGFRs are thought to be involved in critical cellular processes, and induces multiple signal transduction pathways in the cell which have been shown to be up-regulated in many types of cancers. ${ }^{15}$ Of the four FGFR tyrosine kinases, FGFR2 has been reported to be involved in cell proliferation, differentiation, motility, and angiogenesis and dysregulation of this molecule has been associated with cancer development. ${ }^{16,17}$ Regarding HCC, several studies have reported the important roles that disrupted FGFR2 signaling pathways play in HCC progression. An in vivo study of 38 HCC tissues obtained from Hepatitis C virus patients with HCC has reported that FGFR2 is overexpressed in higher T stage than lower $\mathrm{T}$ stage tumors. ${ }^{18}$ Another clinical study of $46 \mathrm{HCC}$ patients who underwent curative hepatectomy has reported that the high FGFR2 expression correlated significantly with a poor histological differentiation, a higher incidence of portal vein invasion, and poor prognosis." Our study of a large cohort of HCC patients could provide evidence to support the clinical implication of FGFR2 expression in HCC by showing that FGFR2 is only expressed in HCC tissues, and that FGFR2 positivity in HCC tissues is associated with poor overall survival rates in the large cohort, though this finding was not proved in the validation set. We intended to validate the result from the development set in an additional, independent HCC cohort. However, only liquid nitrogen-stored sample were available in this cohort, so we had no choice but to use western blot. The discrepancy between the prognostic results of the development set and those of validation set, is probably due to the differences in the evaluation methods for FGFR2 expression, as well as the relatively small number of patients in this additional cohort. Taken together, the evidence from previous reports and our current study indicates that signaling through FGFR2 may contribute to the development or progression of HCC, and may also be a valuable prognostic indicator of survival in this disease. These findings further suggest that the blockade of FGFR2 might offer a potential therapeutic option in HCC.

The established genetics of FGFR2 in cancer include gene amplification, missense mutations, and single nucleotide polymorphisms (SNPs). ${ }^{19}$ Gene amplification and overexpression of FGFR2 are known to occur in human breast cancer ${ }^{20}$ and gastric cancer. ${ }^{21}$ Despite the increasing evidence implicating altered FGFR2 expression in the etiology of HCC, the genetics of FGFR2 and the downstream molecular effects of enhanced FGFR expression in this cancer remain unclear. Further study is warranted to investigate the genet- 
ics of FGFR2 and effects of altered FGFR2 expression on downstream signaling in HCC.

Of the four FGFR tyrosine kinases, FGFR4 is dominant in mature hepatocytes. ${ }^{22}$ The expression of other FGFR isotypes in the liver appears to be limited to nonparenchymal cells and hepatocyte progenitors. ${ }^{23,24}$ Our current findings are that FGFR4 is expressed in most of the non-tumor liver tissues (82.9\%), and interestingly, that FGFR4 expression is reduced significantly in paired HCC tissues. Several previous studies have investigated the role of FGFR4 in HCC, and the FGF19/FGFR4 system is the most studied mechanism in relation to this role. Recently, a report of 40 human HCC tissues showed that FGF19 is expressed at significantly higher levels in HCC, and that FGF19 and FGFR4 inhibition suppresses proliferation and increases apoptosis in HCC cell lines. ${ }^{25}$ In addition, it has been reported also that FGF19/FGFR4-mediated downstream signaling is significantly reduced in tumor samples from mice treated with the FGF19-neutralizing antibody compared with control mice. ${ }^{26}$ These findings suggest that the FGF19/FGFR4 system plays a critical role in HCC progression. However, there are also several reports with contradictory findings. A recent study reported that the genetic deletion of FGFR4 markedly accelerates hepatocarcinogenesis initiated by a single neonatal exposure to the hepatocyte-activated pro-carcinogen diethylnitrosamine, indicating that FGFR4 suppresses hepatoma progression. ${ }^{13,27}$ Although our present finding did not show any evident clinical findings regarding FGFR4 expression in HCC, the difference in FGFR4 expression patterns between HCC and non-tumor liver tissues was a remarkable finding. Therefore, further evaluation to elucidate the exact role of FGFR4 in HCC would be invaluable.

FGFR1 and FGFR3 are also thought to play a role in hepatocarcinogenesis. FGFR1 expression in the liver appears to be limited to non-parenchymal cells and hepatocyte progenitors. ${ }^{23,24}$ However, the ectopic expression of FGFR1 has been proved in HCC and is known to promote the development of HCC in response to carcinogenic stimulation. ${ }^{28}$ FGFR1-mediated signaling is involved in cancer cell growth and infiltration, as well as in angiogenesis. ${ }^{22} \mathrm{~A}$ recent study showed that treatment with a combination of interferon- $\alpha / \beta$ and anti-FGFR1 monoclonal antibody suppresses $\mathrm{HCC}$ cell growth in vitro and in vivo. ${ }^{29}$ In addition, another study using 65 human HCC samples showed that the downregulation of miR-214 in HCC and the upregulation of its target gene FGFR1 is associated with HCC progression. ${ }^{30}$ These studies suggest that FGFR1 could be used as a novel therapeutic target in HCC. FGFR3 is also involved in cell growth control, cell differentiation, and migration. ${ }^{31}$ A recent study using 43 human HCC samples revealed that the expression of FGFR3 increased significantly in HCC, and the overexpression of FGFR3 was correlated with poor tumor differentiation and high nuclear grade. ${ }^{32}$ Therefore, the activation of FGFR3 may play an important role in hepatocarcinogenesis. Two studies reported the overexpression of FGFR1 and FGFR3 in HCC compared with non-neoplastic liver tissue using human tissues, but they involved a relatively small number of samples ( $n=65$ and $n=43$, respectively). ${ }^{30,32}$ Hence, the true prevalence of the overexpression of FGFR1 and FGFR3 in the general HCC population is unknown to date. The present study which used a large HCC cohort showed that FGFR1 and FGFR3 were expressed in only a very small number of early-stage HCCs; this finding is different from the results of previous studies. ${ }^{30,32}$ These discrepancies could be explained by various conditions, including different antibodies, and differences in the analysis methods used and the number of samples or the heterogeneous HCC population. We speculate that although FGFR1 and FGFR3 have some role in hepatocarcinogenesis, they might only act in limited circumstances of patients or specific stages of carcinogenesis. However, it is still difficult to draw a definitive conclusion; additional larger studies, including those on advanced HCC, are needed to identify the roles of FGFR1 and FGFR3, accompanied by research to determine their mechanisms.

Our present analysis has several limitations to note. The tissue samples used in our experiments were obtained from HCC patients who underwent curative resection. Hence, our data are unlikely to reflect the clinical outcomes of advanced HCC because the patients included in our study cohort had relatively early stage HCCs. FGFRs are thought to be involved in various steps of carcinogenesis and the FGFR expression pattern of advanced HCC and early HCC may well be different. Another limitation is that we did not evaluate the pathological characteristics of neighboring liver tissues. The FGF/FGFR signaling axis is also a contributor to fibrosis in the liver, ${ }^{33,34}$ the FGFR expression profile of neighboring liver tissues may be associated with the fibrosis level. The difference of the evaluation methods for FGFR2 expression between the development set and the validation set is also one of limitation.

Nevertheless, our current data have important clinical implications for HCC because we have reported the first large scale analysis of FGFR isotype expression and the prognostic implications of this for HCC patients. Our data on the FGFR isotype expression profile in human liver tissues and its relationship to clinical outcomes might provide a valuable basis for the future development of novel HCC therapies, particularly molecular targeted therapies. In conclusion, positive FGFR2 expression in HCC tissues could be 
Hyo Jeong Lee, et al. FGFR2 expression predicts survival in HCC

an independent prognostic marker of postsurgical survival in HCC patients. Hence, FGFR2 expression in HCC has potential utility as a prognostic biomarker for HCC patients after hepatic resection, and as a novel molecular therapeutic target for this disease.

\section{Acknowledgements}

This study was supported by a grant from AstraZeneca Pharmaceuticals and Yuhan corporation.

\section{Conflicts of Interest}

The authors have no conflicts to disclose.

\section{SUPPLEMENTARY MATERIALS}

Supplementary data related to this article is available online http://e-cmh.org/suppl/chm-21-1-60-s001.pdf.

\section{REFERENCES}

1. Llovet JM, Burroughs A, Bruix J. Hepatocellular carcinoma. The Lancet 2003;362:1907-1917.

2. Sherman M. Hepatocellular carcinoma: Epidemiology, surveillance, and diagnosis. Semin Liver Dis 2010;30:3-16.

3. Ho CM, Lee PH, Shau WY, Ho MC, Wu YM, Hu RH. Survival in patients with recurrent hepatocellular carcinoma after primary hepatectomy: Comparative effectiveness of treatment modalities. Surgery 2012;5:700-709.

4. Zhong JH, Li H, Li LQ, You XM, Zhang Y, Zhao YN, et al. Adjuvant therapy options following curative treatment of hepatocellular carcinoma: A systematic review of randomized trials. Eur J Surg Oncol 2012;38:286-295.

5. Llovet JM, Bustamante J, Castells A, Vilana R, Ayuso MDC, Sala M, et al. Natural history of untreated nonsurgical hepatocellular carcinoma: Rationale for the design and evaluation of therapeutic trials. Hepatology 1999;29:62-67.

6. Natsuizaka M, Omura T, Akaike T, Kuwata Y, Yamazaki K, Sato T, et al. Clinical features of hepatocellular carcinoma with extrahepatic metastases. J Gastroenterol Hepatol 2005;20:1781-1787.

7. Llovet JM, Ricci S, Mazzaferro V, Hilgard P, Gane E, Blanc JF, et al. Sorafenib in advanced hepatocellular carcinoma. N Engl J Med 2008;359:378-390.

8. Llovet JM, Bruix J. Molecular targeted therapies in hepatocellular carcinoma. Hepatology 2008:48:1312-1327.

9. Turner N, Grose R. Fibroblast growth factor signalling: From develop- ment to cancer. Nat Rev Cancer 2010;10:116-129.

10. Amann T, Bataille F, Spruss T, Dettmer K, Wild P, Liedtke $C$, et al. Reduced expression of fibroblast growth factor receptor 2iiib in hepatocellular carcinoma induces a more aggressive growth. Am J Pathol 2010;176:1433-1442.

11. Harimoto N, Taguchi K, Shirabe K, Adachi E, Sakaguchi Y, Toh Y, et al. The significance of fibroblast growth factor receptor 2 expression in differentiation of hepatocellular carcinoma. Oncology 2010;78:361368.

12. Ho HK, Pok S, Streit S, Ruhe JE, Hart S, Lim KS, et al. Fibroblast growth factor receptor 4 regulates proliferation, anti-apoptosis and alphafetoprotein secretion during hepatocellular carcinoma progression and represents a potential target for therapeutic intervention. J Hepatol 2009;50:118-127.

13. Huang X, Yang C, Jin C, Luo Y, Wang F, McKeehan WL. Resident hepatocyte fibroblast growth factor receptor 4 limits hepatocarcinogenesis. Mol Carcinog 2009;48:553-562.

14. Gauglhofer C, Sagmeister S, Schrottmaier W, Fischer C, RodgarkiaDara C, Mohr T, et al. Up-regulation of the fibroblast growth factor 8 subfamily in human hepatocellular carcinoma for cell survival and neoangiogenesis. Hepatology 2011;53:854-864.

15. Kwabi-Addo B, Ozen M, Ittmann M. The role of fibroblast growth factors and their receptors in prostate cancer. Endocr Relat Cancer 2004:11:709-724.

16. Grose R, Dickson C. Fibroblast growth factor signaling in tumorigenesis. Cytokine Growth Factor Rev 2005;16:179-186.

17. Ingersoll RG, Paznekas WA, Tran AK, Scott AF, Jiang G, Jabs EW. Fibroblast growth factor receptor 2 (fgfr2): Genomic sequence and variations. Cytogenet Cell Genet 2001;94:121-126.

18. Mas VR, Maluf DG, Archer KJ, Yanek KC, Fisher RA. Angiogenesis soluble factors as hepatocellular carcinoma noninvasive markers for monitoring hepatitis c virus cirrhotic patients awaiting liver transplantation. Transplantation 2007;84:1262-1271.

19. Katoh M. Cancer genomics and genetics of fgfr2 (review). Int J Oncol 2008:33:233-237.

20. Adnane J, Gaudray P, Dionne CA, Crumley G, Jaye M, Schlessinger J, et al. Bek and flg, two receptors to members of the fgf family, are amplified in subsets of human breast cancers. Oncogene 1991;6:659-663.

21. Katoh M. Fgf signaling network in the gastrointestinal tract (review). Int J Oncol 2006;29:163-168.

22. Kan M, Wu X, Wang F, McKeehan WL. Specificity for fibroblast growth factors determined by heparan sulfate in a binary complex with the receptor kinase. J Biol Chem 1999;274:15947-15952.

23. Hu Z, Evarts RP, Fujio K, Marsden ER, Thorgeirsson SS. Expression of fibroblast growth factor receptors flg and bek during hepatic ontogenesis and regeneration in the rat. Cell Growth Differ 1995;6:1019-1025.

24. Hu Z, Evarts RP, Fujio K, Omori N, Omori M, Marsden ER, et al. Expression of transforming growth factor alpha/epidermal growth factor 
receptor, hepatocyte growth factor/c-met and acidic fibroblast growth factor/fibroblast growth factor receptors during hepatocarcinogenesis. Carcinogenesis 1996;17:931-938.

25. Miura S, Mitsuhashi N, Shimizu H, Kimura F, Yoshidome H, Otsuka M, et al. Fibroblast growth factor 19 expression correlates with tumor progression and poorer prognosis of hepatocellular carcinoma. BMC Cancer 2012;12:56.

26. Desnoyers LR, Pai R, Ferrando RE, Hötzel K, Le T, Ross J, et al. Targeting fgf19 inhibits tumor growth in colon cancer xenograft and fgf19 transgenic hepatocellular carcinoma models. Oncogene 2008;27:8597.

27. Luo Y, Yang C, Lu W, Xie R, Jin C, Huang P, et al. Metabolic regulator betaklotho interacts with fibroblast growth factor receptor 4 (fgfr4) to induce apoptosis and inhibit tumor cell proliferation. J Biol Chem 2010;285:30069-30078.

28. Huang $X$, Yu C, Jin C, Kobayashi M, Bowles CA, Wang F, et al. Ectopic activity of fibroblast growth factor receptor 1 in hepatocytes accelerates hepatocarcinogenesis by driving proliferation and vascular endothelial growth factor-induced angiogenesis. Cancer Res 2006;66:1481-
1490.

29. Sasaki S, Ishida T, Toyota M, Ota A, Suzuki H, Takaoka A, et al. Interferon-alpha/beta and anti-fibroblast growth factor receptor 1 monoclonal antibody suppress hepatic cancer cells in vitro and in vivo. PLoS One 2011;6:e19618.

30. Wang J, Li J, Wang X, Zheng C, Ma W. Downregulation of microrna-214 and overexpression of fgfr-1 contribute to hepatocellular carcinoma metastasis. Biochem Biophys Res Commun 2013;439:47-53.

31. Kanai M, Göke M, Tsunekawa S, Podolsky DK.. Signal transduction pathway of human fibroblast growth factor receptor 3. Identification of a novel 66-kda phosphoprotein. J Biol Chem 1997;272:6621-6628.

32. Qiu WH, Zhou BS, Chu PG, Chen WG, Chung C, Shih J, et al. Overexpression of fibroblast growth factor receptor 3 in human hepatocellular carcinoma. World J Gastroenterol 2005;11:5266-5272.

33. Bataller R, Brenner DA. Liver fibrosis. J Clin Invest 2005;115:209-218.

34. Gressner AM, Weiskirchen R. Modern pathogenetic concepts of liver fibrosis suggest stellate cells and tgf-beta as major players and therapeutic targets. J Cell Mol Med 2006;10:76-99. 